\title{
Une analyse contextuelle des indicateurs de surveillance du suicide
}

\author{
Robin Skinner, M.S.P.; Brittany Irvine, M.A.; Gabriela Willams, M. Sc.; Caryn Pearson, M.A.; Jaskiran Kaur, B. Sc. S.; \\ Xiaoquan Yao, M. Sc.; Lee Merklinger, M.A.; Tanya Lary, M.A.
}

Diffuser cet article sur Twitter

Le Cadre fédéral de prévention du suicide ${ }^{1}$ a été élaboré par l'Agence de la santé publique du Canada (ASPC) afin d'établir l'approche stratégique du Canada en matière de prévention du suicide, conformément à la Loi concernant l'établissement d'un cadre fédéral de prévention du suicide (le "Cadre »), qui a été promulguée en décembre 2012. Le Cadre incluait un engagement à présenter les statistiques sur le suicide et les facteurs de risques connexes au public. Dans le cadre de cet engagement, l'ASPC a mis au point un ensemble d'indicateurs nécessaires à la surveillance globale du suicide visant à éclairer les initiatives de prévention du suicide. Ces indicateurs fournissent des mesures des résultats ainsi que des facteurs de risque et de protection aux niveaux personnel, familial, communautaire et sociétal.

Les indicateurs de surveillance du suicide (ISS) ont été sélectionnés au moyen d'un examen de la documentation pertinente extraite de certaines bases de données de publications (p. ex. PubMed) et des restrictions sur le type de preuve ont été appliquées : seuls les revues systématiques, les méta-analyses, les revues de la littérature ou les rapports ministériels, publiés en anglais ou en français dans les six dernières années, étaient admissibles. On a déterminé des indicateurs (et des sources de données pour opérationnaliser les indicateurs) à partir de cet ensemble de preuves. On a demandé à des intervenants internes et externes de formuler leurs commentaires sur les indicateurs et leur opérationnalisation. Il est important de noter que la revue de la littérature ne visait que la population générale.

Cet aperçu propose les plus récentes statistiques disponibles pour établir des rapports sur les ISS. De plus, il présente une brève analyse des éléments des ISS sélectionnés qui révèle l'importance des associations entre certaines maladies mentales ${ }^{2,3}$ et certains comportements suicidaires (idées suicidaires, plans ou tentatives de suicide) à l'aide de l'Enquête sur la santé dans les collectivités canadiennes - Santé mentale de 2012 (ESCC-SM) ${ }^{4}$. De nouvelles études devraient continuer d'explorer et de surveiller l'association entre le taux de suicide et les autres facteurs de risque et de prévention (p. ex. la violence faite aux enfants, le soutien social) ${ }^{5}$ énumérés dans les ISS afin d'utiliser ces connaissances dans le cadre des initiatives de prévention du suicide.

\section{Résultats et discussion}

Le tableau 1 présente les statistiques rapides et les taux d'incidence canadiens actuels de décès liés au suicide, d'hospitalisations associées à des blessures auto-infligées et de visites à l'urgence, ainsi que la prévalence des idées suicidaires, des plans et des tentatives de suicide, de même qu'une liste des principaux facteurs de risque et de protection. Les statistiques sur l'état civil du Canada indiquent que le taux de décès liés au suicide était de 11,5 pour 100000 personnes en 2013. Les données de l'ESCC 2015 indiquent que $2,5 \%$ des Canadiens de 15 ans ou plus ont déclaré avoir eu des idées suicidaires (c.-à-d. qu'ils envisagent sérieusement le suicide), 0,8\% ont déclaré avoir élaboré un plan, et $0,4 \%$ ont déclaré avoir fait une tentative de suicide au cours des 12 derniers mois.

En ce qui concerne les maladies mentales, les données de l'ESCC-SM 2012 indiquent que $6,5 \%$ (IC à $95 \%: 6,0$ à 7,0) des Canadiens de 15 ans et plus ont éprouvé des symptômes associés à un trouble de l'humeur ou à un trouble d'anxiété généralisée au cours des 12 derniers mois, 1,3\% (IC à $95 \%: 1,1$ à 1,5 ) ont déclaré avoir déjà reçu un diagnostic de schizophrénie ou de psychose par un professionnel de la santé, et $1,7 \%$ (IC à $95 \%: 1,4$ à 2,0 ) ont déclaré avoir déjà reçu un diagnostic de trouble de stress post-traumatique.

Le tableau 2 présente des rapports de cotes (RC) non corrigés entre les maladies mentales et les comportements suicidaires sélectionnés calculés à partir de l'ESCCSM. Lors de cette phase d'analyse, l'objectif était d'évaluer les relations individuelles avec le suicide; reste à contrôler les facteurs de confusion. De plus, les ventilations des proportions selon des variables telles que l'âge et le sexe ne seront disponibles qu'ultérieurement sur l'infobase de la santé publique du gouvernement du Canada. Conformément à la littérature, le fait d'éprouver des symptômes associés à un trouble de l'humeur ou à un trouble d'anxiété généralisée s'est révélé fortement associé aux comportements suicidaires $(\mathrm{RC}=18,1$, IC à $95 \%: 14,3$ à 23,0). De plus, le fait de déclarer avoir reçu un diagnostic de schizophrénie/psychose, de trouble de stress post-traumatique ou de trouble alimentaire était également fortement associé aux comportements suicidaires, avec des RC de 10,6 (IC à $95 \%$ : 6,9 à $16,1), 15,5$ (IC à $95 \%: 10,3$ à 23,5) et 13,0 (IC à $95 \%: 5,6$ à 30,3) respectivement.

Le contrôle et la divulgation en continu des données de surveillance sur les décès liés aux suicides, les comportements suicidaires et les facteurs de risque et de protection connexes constituent un élément fondamental de la prévention du suicide au Canada. 


\section{INDICATEURS DE LA SURVEILLANCE DU SUICIDE}

\section{STATISTIQUES RAPIDES, CANADA, ÉDITION 2017}

\begin{tabular}{|c|c|c|c|}
\hline GROUPE D'INDICATEURS & MESURE(S) D'INDICATEUR & $\begin{array}{l}\text { ESTIMATION LA PLUS } \\
\text { RÉCENTE }\end{array}$ & $\begin{array}{l}\text { SOURCE DE } \\
\text { DONNÉES (ANNÉE) }\end{array}$ \\
\hline \multicolumn{4}{|c|}{ SUICIDE ET BLESSURES AUTO-INFLIGÉES - CARACTÉRISTIQUES } \\
\hline Taux de mortalité par suicide & $\begin{array}{l}\text { Taux de mortalité au sein de l'ensemble de la population attribuable au } \\
\text { suicide }\end{array}$ & $\begin{array}{r}11,5 \text { pour } \\
100000 \text { habitants }\end{array}$ & SEC (2013) \\
\hline $\begin{array}{l}\text { Blessures auto-infligées, } \\
\text { hospitalisations }\end{array}$ & $\begin{array}{l}\text { Taux d'hospitalisation en raison de blessures auto-infligées (à l'exception } \\
\text { du Québec) }\end{array}$ & $\begin{array}{r}50,2 \text { hospitalisations pour } \\
100000 \text { habitants }\end{array}$ & BDCP (2014-2015) \\
\hline \multirow[t]{3}{*}{$\begin{array}{l}\text { Blessures auto-infligées, } \\
\text { présentations à l'urgenence }\end{array}$} & $\begin{array}{l}\text { Taux de présentation à l'urgence en raison de blessures auto-infligées } \\
\text { (Ontario) }\end{array}$ & $\begin{array}{l}113,9 \text { visites à l'urgence } \\
\text { pour } 100000 \text { habitants }\end{array}$ & $\begin{array}{r}\text { SNISA (2014-2015) } \\
\text { (Ontario) }\end{array}$ \\
\hline & $\begin{array}{l}\text { Taux de présentation à l'urgence en raison de blessures auto-infligées } \\
\text { (Alberta) }\end{array}$ & $\begin{array}{l}160,5 \text { visites à l'urgence } \\
\text { pour } 100000 \text { habitants }\end{array}$ & $\begin{array}{r}\text { SNISA (2014-2015) } \\
\text { (Alberta) }\end{array}$ \\
\hline & $\begin{array}{l}\text { Proportion de présentations à l'urgence en raison de blessures auto- } \\
\text { infligées, sur le total de cas dans l'e-SCHIRPT, chez les } 10 \text { à } 24 \text { ans }\end{array}$ & $\begin{array}{r}1072,8 \text { visites à l'urgence pour } \\
100000 \text { cas dans e-SCHIRPT }\end{array}$ & $\begin{array}{r}\text { e-SCHIRPT } \\
(2011-2016)\end{array}$ \\
\hline \multirow[t]{2}{*}{ Pensées suicidaires } & $\begin{array}{l}\text { \% de la population âgée de } 15 \text { ans et plus ayant déclaré avoir déjà eu } \\
\text { de fortes idées suicidaires (au cours de la vie) }\end{array}$ & $12,3 \%$ & ESCC (2015) \\
\hline & $\begin{array}{l}\text { \% de la population âgée de } 15 \text { ans et plus ayant déclaré avoir eu de fortes } \\
\text { idées suicidaires au cours des } 12 \text { derniers mois }\end{array}$ & $2,5 \%$ & ESCC (2015) \\
\hline \multirow[t]{2}{*}{ Plans de suicide } & $\begin{array}{l}\text { \% de la population âgée de } 15 \text { ans et plus ayant déclaré avoir déjà planifié } \\
\text { de se suicider (au cours de la vie) }\end{array}$ & $4,5 \%$ & ESCC (2015) \\
\hline & $\begin{array}{l}\text { \% de la population âgée de } 15 \text { ans et plus ayant déclaré avoir planifié de se } \\
\text { suicider au cours des } 12 \text { derniers mois }\end{array}$ & $0,8 \%$ & ESCC (2015) \\
\hline \multirow[t]{2}{*}{ Tentatives de suicide } & $\begin{array}{l}\text { \% de la population âgée de } 15 \text { ans et plus ayant déclaré avoir déjà tenté de } \\
\text { se suicider (au cours de la vie) }\end{array}$ & $3,4 \%$ & ESCC (2015) \\
\hline & $\begin{array}{l}\text { \% de la population âgée de } 15 \text { ans et plus ayant déclaré avoir tenté de se } \\
\text { suicider au cours des } 12 \text { derniers mois }\end{array}$ & $0,4 \%$ & ESCC (2015) \\
\hline \multicolumn{4}{|c|}{ FACTEURS DE RISOUE ET DE PROTECTION - INDIVIDU } \\
\hline \multirow[t]{4}{*}{ État de santé } & $\begin{array}{l}\text { \% de la population âgée de } 12 \text { ans et plus déclarant avoir une santé } \\
\text { «passable » ou «mauvaise» }\end{array}$ & $10,7 \%$ & ESCC (2015) \\
\hline & $\begin{array}{l}\% \text { de la population âgée de } 12 \text { ans et plus déclarant avoir une santé } \\
\text { mentale «passable» ou «mauvaise } »^{a}\end{array}$ & $5,9 \%$ & ESCC (2015) \\
\hline & $\begin{array}{l}\text { \% de la population âgée de } 12 \text { ans et plus atteinte d'une incapacité } \\
\text { modérée ou grave, selon l'IES }{ }^{b}\end{array}$ & $31,7 \%$ & ESCC (2015) \\
\hline & $\begin{array}{l}\text { \% de la population âgée de } 12 \text { ans et plus atteinte d'une ou de plusieurs } \\
\text { des maladies chroniques majeures suivantes : cancer, diabète, maladie } \\
\text { cardiovasculaire ou maladie respiratoire }\end{array}$ & $23,7 \%$ & ESCC (2015) \\
\hline Douleur chronique & $\begin{array}{l}\text { \% de la population âgée de } 12 \text { ans et plus déclarant avoir des douleurs } \\
\text { modérées ou graves qui l'« empêchent de faire quelques-unes» ou «la } \\
\text { plupart» de ses activités }\end{array}$ & $8,3 \%$ & ESCC (2015) \\
\hline Troubles du sommeil & $\begin{array}{l}\text { \% de la population âgée de } 15 \text { ans et plus déclarant avoir des problèmes à } \\
\text { s'endormir ou à demeurer endormi «la plupart du temps» ou «tout le } \\
\text { temps» }\end{array}$ & $14,5 \%$ & $\begin{array}{r}\text { ESCC - Santé mentale } \\
\text { (2012) }\end{array}$ \\
\hline Stress & $\begin{array}{l}\text { \% de la population âgée de } 12 \text { ans et plus déclarant la plupart de ses } \\
\text { journées «assez stressantes» ou «extrêmement stressantes» au cours des } \\
12 \text { derniers mois }\end{array}$ & $21,4 \%$ & ESCC (2015) \\
\hline \multirow[t]{4}{*}{ Maladie mentale } & $\begin{array}{l}\text { \% de la population âgée de } 15 \text { ans et plus déclarant avoir eu des } \\
\text { symptômes correspondant à un trouble de l'humeur ou au trouble } \\
\text { d'anxiété généralisé (TAG) au cours des } 12 \text { derniers mois }\end{array}$ & $6,5 \%$ & $\begin{array}{r}\text { ESCC - Santé mentale } \\
(2012)\end{array}$ \\
\hline & $\begin{array}{l}\text { \% de la population âgée de } 15 \text { ans et plus déclarant avoir reçu un } \\
\text { diagnostic de schizophrénie ou de psychose de la part d'un professionnel } \\
\text { de la santéd }\end{array}$ & $1,3 \%$ & $\begin{array}{r}\text { ESCC - Santé mentale } \\
\text { (2012) }\end{array}$ \\
\hline & $\begin{array}{l}\text { \% de la population âgée de } 15 \text { ans et plus déclarant avoir reçu un } \\
\text { diagnostic de trouble de l'alimentation de la part d'un professionnel de la } \\
\text { santéd }^{\text {la }}\end{array}$ & $0,4 \%$ & $\begin{array}{r}\text { ESCC - Santé mentale } \\
(2012)\end{array}$ \\
\hline & $\begin{array}{l}\text { \% de la population âgée de } 15 \text { ans et plus déclarant avoir reçu un } \\
\text { diagnostic de trouble de stress post-traumatique (TSPT) de la part d'un } \\
\text { professionnel de la santé }\end{array}$ & $1,7 \%$ & $\begin{array}{r}\text { ESCC - Santé mentale } \\
(2012)\end{array}$ \\
\hline \multirow[t]{3}{*}{ Violence } & $\begin{array}{l}\text { \% de la population âgée de } 15 \text { ans et plus déclarant avoir été victime, avant } \\
\text { l'âge de } 15 \text { ans, d'au moins un des actes suivants : violence physique ou } \\
\text { sexuelle par une personne de } 18 \text { ans ou plus, ou exposition à des actes de } \\
\text { violence commis par un parent ou tuteur }\end{array}$ & $32,9 \%$ & $\begin{array}{r}\text { ESG - Victimisation } \\
\text { (2014) }\end{array}$ \\
\hline & $\begin{array}{l}\text { \% de la population âgée de } 15 \text { ans et plus déclarant avoir été victime de } \\
\text { violence physique ou sexuelle au cours des } 12 \text { derniers mois }\end{array}$ & $4,1 \%$ & $\begin{array}{r}\text { ESG - Victimisation } \\
(2014)\end{array}$ \\
\hline & $\begin{array}{l}\text { \% d'élèves de la } 6^{e} \text { à la } 12^{e} \text { année déclarant avoir été victime d'actes } \\
\text { d'intimidation perpétrés par d'autres élèves au cours des } 30 \text { derniers jours }\end{array}$ & $25,1 \%$ & $\begin{array}{r}\text { ECTADE } \\
(2014-2015)\end{array}$ \\
\hline $\begin{array}{l}\text { Consommation d'alcool ou } \\
\text { de drogues }\end{array}$ & $\begin{array}{l}\text { \% de la population âgée de } 15 \text { ans et plus déclarant consommer davantage } \\
\text { d'alcool que ce que préconisent les Directives de consommation d'alcool à } \\
\text { faible risque du Canada }^{\mathrm{e}}\end{array}$ & $15,7 \%$ & ECTAD (2013) \\
\hline
\end{tabular}




\begin{tabular}{|c|c|c|c|}
\hline GROUPE D'INDICATEURS & MESURE(S) D'INDICATEUR & $\begin{array}{l}\text { ESTIMATION LA PLUS } \\
\text { RÉCENTE }\end{array}$ & $\begin{array}{l}\text { SOURCE DE } \\
\text { DONNÉES (ANNÉE) }\end{array}$ \\
\hline Milieu scolaire & $\begin{array}{l}\text { \% d'élèves de la } 6^{e} \text { à la } 10^{e} \text { année déclarant être « d'accord » ou « tout à fait } \\
\text { d'accord » qu'ils éprouvent un sentiment d'appartenance à leur école }\end{array}$ & $63,1 \%$ & $\begin{array}{r}\text { Enquête HBSC } \\
(2013-2014)\end{array}$ \\
\hline \multicolumn{4}{|c|}{ FACTEURS DE RISQUE ET DE PROTECTION - FAMILLE } \\
\hline \multirow[t]{2}{*}{ Relations familiales } & $\begin{array}{l}\% \text { d'élèves de la } 6^{\mathrm{e}} \text { à la } 10^{\mathrm{e}} \text { année déclarant recevoir de leur famille le } \\
\text { soutien émotionnel dont ils ont besoin }\end{array}$ & $64,9 \%$ & $\begin{array}{r}\text { Enquête HBSC } \\
(2013-2014)\end{array}$ \\
\hline & $\begin{array}{l}\% \text { d'élèves de la } 6^{\mathrm{e}} \text { à la } 10^{\mathrm{e}} \text { année déclarant se disputer souvent avec leurs } \\
\text { parents }\end{array}$ & $21,7 \%$ & $\begin{array}{r}\text { Enquête HBSC } \\
(2013-2014)\end{array}$ \\
\hline $\begin{array}{l}\text { Maladies mentales et } \\
\text { consommation d'alcool ou de } \\
\text { drogues au sein de la famille }\end{array}$ & $\begin{array}{l}\text { \% de la population âgée de } 15 \text { ans et plus déclarant être touchée «assez» } \\
\text { ou «beaucoup» par les émotions, les problèmes de santé mentale ou la } \\
\text { consommation d'alcool ou de drogues d'un ou de plusieurs membres de la } \\
\text { famille }\end{array}$ & $13,7 \%$ & $\begin{array}{r}\text { ESCC - Santé mentale } \\
\text { (2012) }\end{array}$ \\
\hline $\begin{array}{l}\text { Antécédents familiaux de } \\
\text { comportement suicidaire }\end{array}$ & \multicolumn{3}{|l|}{ En cours d'élaboration } \\
\hline \multicolumn{4}{|c|}{ FACTEURS DE RISOUE ET DE PROTECTION - COMMUNAUTÉ } \\
\hline \multirow[t]{2}{*}{ Soutien social } & $\begin{array}{l}\text { \% de la population âgée de } 15 \text { ans et plus n'ayant aucun membre de la } \\
\text { famille ou ami intime avec qui elle est à l'aise, à qui elle peut dire ce } \\
\text { qu'elle pense ou à qui elle peut demander de l'aide }\end{array}$ & $6,0 \%$ & $\begin{array}{r}\text { ESG - Identité sociale } \\
\text { (2013) }\end{array}$ \\
\hline & $\begin{array}{l}\text { \% de la population âgée de } 15 \text { ans et plus déclarant être «satisfaite» ou } \\
\text { «très satisfaite» de la fréquence des communications avec des amis ou des } \\
\text { membres de la famille ne vivant pas avec elle }\end{array}$ & $91,5 \%$ & $\begin{array}{r}\text { ESG - Identité sociale } \\
\text { (2013) }\end{array}$ \\
\hline $\begin{array}{l}\text { Sentiment d'appartenance à } \\
\text { la communauté }\end{array}$ & $\begin{array}{l}\text { \% de la population âgée de } 12 \text { ans et plus déclarant éprouver un sentiment } \\
\text { d'appartenance «plutôt fort» ou «très fort» à la collectivité locale }\end{array}$ & $67,9 \%$ & ESCC (2015) \\
\hline $\begin{array}{l}\text { Suicide par imitation/série de } \\
\text { suicides }\end{array}$ & \multicolumn{3}{|l|}{ En cours d'élaboration } \\
\hline \multicolumn{4}{|c|}{ FACTEURS DE RISQUE ET DE PROTECTION - SOCIÉTÉ } \\
\hline \multirow[t]{2}{*}{$\begin{array}{l}\text { Accès aux services en matière } \\
\text { de santé mentale }\end{array}$} & $\begin{array}{l}\text { \% de la population âgée de } 15 \text { ans et plus qui estime avoir eu besoin de } \\
\text { soins de santé mentale au cours des } 12 \text { derniers mois }\end{array}$ & $17,5 \%$ & $\begin{array}{r}\text { ESCC - Santé mentale } \\
\text { (2012) }\end{array}$ \\
\hline & $\begin{array}{l}\text { \% de la population âgée de } 15 \text { ans et plus qui estime avoir eu besoin de } \\
\text { soins de santé mentale au cours des } 12 \text { derniers mois, mais dont le besoin } \\
\text { n'aurait pas été comblé ou ne l'aurait été qu'en partie }\end{array}$ & $33,3 \%$ & $\begin{array}{r}\text { ESCC - Santé mentale } \\
\text { (2012) }\end{array}$ \\
\hline Possession d'armes à feu & $\begin{array}{l}\text { Nombre de détenteurs d'un permis d'arme à feu pour particulier de } 18 \text { ans } \\
\text { et plus en date de décembre } 2016 \text { (\% de la population âgée de } 18 \text { ans et } \\
\text { plus qui détient un permis d'arme à feu) }\end{array}$ & $2066961(7,3 \%)$ & GRC (2016) \\
\hline $\begin{array}{l}\text { Connaissances en santé } \\
\text { mentale }\end{array}$ & \multicolumn{3}{|l|}{ En cours d'élaboration } \\
\hline $\begin{array}{l}\text { Lignes directrices sur la } \\
\text { couverture médiatique } \\
\text { (conformité) }\end{array}$ & \multicolumn{3}{|l|}{ En cours d'élaboration } \\
\hline Stigmatisation & \multicolumn{3}{|l|}{ En cours d'élaboration } \\
\hline $\begin{array}{l}\text { Contrôle des } \\
\text { empoisonnements }\end{array}$ & \multicolumn{3}{|l|}{ En cours d'élaboration } \\
\hline
\end{tabular}

Abréviations : BDCP, Base de données sur les congés des patients; CV, coefficient de variation; ECI, Étude canadienne sur l'incidence des signalements de cas de violence et de négligence envers les enfants; ECTAD, Enquête canadienne sur le tabac, l'alcool et les drogues; ECTADE, Enquête canadienne sur le tabac, l'alcool et les drogues chez les élèves; ESCC, Enquête sur la santé dans les collectivités canadiennes; e-SCHIRPT, base de données électronique du Système canadien hospitalier d'information et de recherche en prévention des traumatismes; ESG, Enquête sociale générale; GRC, Gendarmerie royale du Canada; HBSC, comportements de santé des jeunes d'âge scolaire; SCSMC, Système canadien de surveillance des maladies chroniques; SEC, Statistique de l'état civil; SNISA, Système national d'information sur les soins ambulatoires.

Remarques : Les catégories entre guillemets s'inscrivent dans une échelle à cinq niveaux (sauf dans le cas de l'indicateur « Sentiment d'appartenance à la communauté », pour lequel une échelle à quatre niveaux a été utilisée). « En cours d'élaboration » concerne les indicateurs dont la source de données est actuellement indisponible et ceux pour lesquels d'autres recherches sont nécessaires afin d'en déterminer une mesure et une source de données satisfaisantes.

Les estimations dont le CV est inférieur à 16,6 \% sont considérées comme fiables et peuvent être utilisées. Les estimations dont le CV se situe entre 16,6\% et 33,3\% doivent être interprétées avec prudence en raison de taux d'erreur élevés (E). Les estimations dont le CV est supérieur à 33,3\% ne sont pas incluses car on considère qu'elles ne sont pas fiables ( ()$^{6}$.

${ }^{a}$ Comprendre ce qui constitue une santé mentale positive pourrait aider les efforts de prévention du suicide. Pour obtenir des renseignements sur la mesure des caractéristiques positives en matière de santé mentale ainsi que sur les facteurs de risque et de protection associés à ces caractéristiques, en particulier la spiritualité et la capacité d’adaptation, veuillez vous reporter au Cadre d'indicateurs de surveillance de la santé mentale positive : http://infobase.phac-aspc.gc.ca/positive-mental-health/index-fr.aspx

${ }^{\mathrm{b}}$ Indice de l'état de santé (IES).

'Bronchite chronique, emphysème ou maladie pulmonaire obstructive chronique.

d Maladies diagnnostiquées par un professionnel de la santé et qui durent depuis au moins 6 mois ou dont on s'attend à qu'elles durent au moins 6 mois.

e Les lignes directrices recommandées sont les suivantes : « au plus 2 verres par jour ou 10 verres par semaine pour les femmes, et au plus 3 verres par jour ou 15 verres par semaine pour les hommes, avec éventuellement un verre de plus lors d'occasions spéciales » (Centre canadien sur les dépendances et l'usage de substances). En ligne à : http://www.ccsa.ca/fra/topics/alcohol /drinking-guidelines/pages/default.aspx

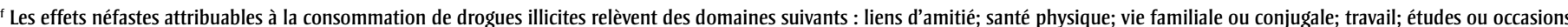
d'emploi; situation financière; rapports avec le système judiciaire; logement et enfin apprentissage.

${ }^{8}$ Gendarmerie royale du Canada. Rapport du commissaire aux armes à feu. 2016. (n ${ }^{0}$ de catalogue : PS96F-PDF). En ligne à : http://www.rcmp-grc.gc.ca/fr/rapport-du-commissaire-aux -armes-a-feu-2016

Citation suggérée : Agence de la santé publique du Canada. Indicateurs de la surveillance du suicide : Statistiques rapides, Canada, édition 2017. Ottawa (Ontario) : Agence de la santé publique du Canada; 2017

Visitez le site des Indicateurs de la surveillance du suicide : http://infobase.phac-aspc.gc.ca/ 
TABLEAU 2

Prévalence des maladies mentales et des comportements suicidaires sélectionnés et associations bidimensionnelles qui les regroupent, estimation pour les Canadiens de $\mathbf{1 5}$ ans et plus

\begin{tabular}{|c|c|c|c|c|c|c|c|}
\hline \multirow{2}{*}{$\begin{array}{l}\text { Facteur } \\
\text { de risque }\end{array}$} & \multirow{2}{*}{$\begin{array}{l}\text { n non } \\
\text { pondéréa }\end{array}$} & \multirow{2}{*}{$\begin{array}{l}\text { Estimation } \\
(\%)\end{array}$} & \multirow{2}{*}{$\begin{array}{c}\text { IC à } 95 \% \text { b', } \\
\alpha=0,05\end{array}$} & \multicolumn{4}{|c|}{ Comportements suicidaires $^{c}$} \\
\hline & & & & $\begin{array}{l}\text { Estimation }^{\mathrm{b}} \\
(\%)\end{array}$ & $\begin{array}{c}\text { IC à } 95 \% \text {, } \\
\alpha=0,05\end{array}$ & $\begin{array}{l}\mathrm{RC}^{\mathrm{b}} \text { non } \\
\text { corrigés }\end{array}$ & $\begin{array}{c}\text { IC à } 95 \% \text {, } \\
\alpha=0,05\end{array}$ \\
\hline \multicolumn{8}{|c|}{$\begin{array}{l}\text { Symptômes associés à un trouble de l'humeur ou à un trouble d'anxiété généralisée au cours des } \\
12 \text { derniers mois }\end{array}$} \\
\hline Oui & 1748 & 6,5 & $6,0-7,0$ & 25,5 & $22,0-29,1$ & 18,1 & \multirow{2}{*}{$14,3-23,0$} \\
\hline Non ${ }^{*}$ & 21876 & 93,5 & $93,0-94,0$ & 1,9 & $1,6-2,1$ & 1,0 & \\
\hline
\end{tabular}

Déclaration d'un diagnostic de schizophrénie ou de psychose par un professionnel de la santé

$\begin{array}{lrrrrrrr}\text { Oui } & 381 & 1,3 & 1,1-1,5 & 25,4 & 17,8-33,1 & 10,6 & 6,9-16,1 \\ \text { Non }^{*} & 23268 & 98,7 & 98,5-99,0 & 3,1 & 2,8-3,5 & 1,0 & \end{array}$

Déclaration d'un diagnostic de trouble de stress post-traumatique par un professionnel de la santé

\begin{tabular}{|c|c|c|c|c|c|c|c|}
\hline Oui & 425 & 1,7 & $1,4-2,0$ & 31,8 & $23,1-40,4$ & 15,5 & \multirow{2}{*}{$10,3-23,5$} \\
\hline Non* & 23205 & 98,3 & $98,1-98,6$ & 2,9 & $2,6-3,2$ & 1,0 & \\
\hline \multicolumn{8}{|c|}{ Déclaration d'un diagnostic de trouble alimentaire par un professionnel de la santé } \\
\hline Oui & 102 & 0,4 & $0,3-0,5$ & $30,7^{\mathrm{E}}$ & $14,1-47,2$ & 13,0 & \multirow{2}{*}{$5,6-30,3$} \\
\hline Non* & 23546 & 99,6 & $99,5-99,7$ & 3,3 & 2,9-3,6 & 1,0 & \\
\hline
\end{tabular}

Source : Enquête sur la santé dans les collectivités canadiennes - Santé mentale de 2012 (partage de données réglementé de Statistique Canada).

Abréviations : IC, intervalle de confiance; RC, rapport de cotes.

Remarque : Les estimations dont le coefficient de variation (CV) est inférieur à 16,6\% sont considérées comme fiables et peuvent être utilisées. Les estimations dont le CV se situe entre 16,6 \% et 33,3\% doivent être interprétées avec prudence en raison de taux d'erreur élevés (E). Les estimations dont le CV est supérieur à 33,3\% ne sont pas incluses car on considère qu'elles ne sont pas fiables $(\mathbf{0})^{6}$.

${ }^{a}$ Le dénombrement inclut seulement les cas où les répondants avaient répondu aux questions du sondage portant sur les comportements suicidaires et à celles portant sur les maladies mentales.

${ }^{\mathrm{b}}$ Les estimations, les IC et les RC sont tous pondérés.

' Les comportements suicidaires incluent les idées suicidaires, les plans et les tentatives de suicide au cours des 12 derniers mois.

*Catégorie de référence pour la mesure des associations.

\section{Références}

1. Agence de la santé publique du Canada. Travailler ensemble pour prévenir le suicide au Canada : Cadre fédéral de prévention du suicide. Ottawa (Ont.) : gouvernement du Canada; 2016 [no de catalogue : HP35 -61/2015E-PDF]. 51 pages.

2. Li Z, Page A, Martin G, Taylor R. Attributable risk of psychiatric and socio-economic factors for suicide from individual level, populationbased studies: a systematic review. Soc Sci Med. 2011;72(4):608-616.

3. Taylor PJ, Hutton P, Wood L. Are people at risk of psychosis also at risk of suicide and self-harm? A systematic review and meta-analysis. Psychol Med. 2015;45(5):911-926.

4. Statistique Canada. Enquête sur la santé dans les communautés canadiennes-Santé mentale de 2012. Ottawa (Ont.) : Statistique Canada; 2013.

5. Afifi TO, MacMillan HL, Boyle M, Taillieu T, Cheung K, Sareen J. Child abuse and mental disorders in Canada. JAMC. 2014;186(9):E324-E332.

6. Statistique Canada. 5.0 Data accuracy and quality [Internet]. Ottawa (Ont.) : Statistique Canada; [modifié le 21 septembre 2009; consulté le 11 mai 2017]. En ligne à : http://www.statcan.gc.ca /pub/13f0026m/2007001/ch5-fra.htm 